(28\%), the media (24\%), and other healthcare providers (16\%). Fifty-four percent of older adult respondents who use cannabis do so with a prescription or medical authorization from their physician / nurse practitioner for medical / therapeutic reasons. One quarter of respondents indicated they use cannabis for non-medical reasons (for recreational use).

Although there is a reported gap in knowledge regarding cannabis and older adults, physicians, nurse practitioners, other healthcare providers and healthcare students all reported they are eager to learn more about how to talk with patients, how to authorize and prescribe cannabis appropriately, how to mitigate risks and assess for cannabis use disorder in older adults. CCSMH will be launching a physicianaccredited e-learning course on Cannabis and Older Adults in January 2022.

\title{
402 - An audit of Lithium Prescribing Practices in an Old Age PsychiatryService highlighting renal impairment in this cohort.
}

Authors: L.Valentine, J.Cannon, S.Marmion, M.Corcoran, M.Cryan, G.Mc Carthy, C.Dolan.

This abstract was presented at RCPsych international congress 2021 and abstract published In special online supplement of BJPsych Open supplement 21-24 June this year 2021-volume 7-Supplement S1 June 2021.

\section{Abstract}

Aims:

To compare Lithium prescribing practices in a Psychiatry of Old Age (POA) Service in the North-West oflreland among adults aged 65 years and over with best practice guidelines.

\section{Methods:}

Review of the literature informed development of audit standards for Lithium prescribing. These included National Institute for Clinical Excellent (NICE) 2014 guidelines, The British National Formulary(2019) and Maudsley Prescribing Guidelines (2018). Data was collected retrospectively, using an audit-specific data collection tool, from clinical files of POA team caseload, aged 65 years or more and prescribed Lithium over the past year.

\section{Results:}

At the time of audit in February 2020, 18 patients were prescribed lithium, 67\% female, average age 74.6 years. Of those prescribed Lithium; $50 \%(n=9)$ had depression, $44 \%(n=8)$ had bipolar affective disorder (BPAD) and 6\% $(n=1)$ schizoaffective disorder.

$78 \%(n=14)$ of patients met the NICE standard of 3-monthly lithium level. Lithium levels were checkedon average 4.5 times in past year, average lithium level was $0.61 \mathrm{mmol} / \mathrm{L}$ across the group and $39 \%$ (n

$=7$ ) had lithium level within recommended therapeutic range $(0.6-0.8 \mathrm{mmol} / \mathrm{L})$.

$83 \%$ of patients $(n=15)$ met the NICE standards of 3 monthly renal tests. Taking into consideration mostrecent blood test results, $100 \%(n=18)$ had abnormal renal function.

Half $(n=9)$ were initiated on lithium by POA service and of these, $56 \%(n=5)$ had documented renal impairment prior to initiation. Of patients on long term lithium at time of referral $(n=9)$, almost half $(n=4)$ had a documented history of lithium toxicity. 


\title{
Conclusions:
}

The results of this audit highlight room for improvement in lithium monitoring of older adults attending POA service. Furthermore, all patients prescribed lithium had impaired renal function. This is an important finding given the associations between those admitted to hospital with COVID-19 and comorbid kidney disease and increased risk of inpatient death.

Our findings highlight the need for three monthly renal function monitoring in elderly prescribed lithiumgiven the additive adverse effects of increasing age and lithium on the kidney. Close working with specialised renal services to provide timely advice on renal management for those with renal impairment prescribed lithium is important to minimise adverse patient outcomes.

\section{3 - Episodes of lucidity (paradoxical lucidity): A survey of family caregivers of persons with dementia}

Presenter: Joan M. Griffin, Ph.D.

\section{Co-authors:}

Kyungmin Kim, PhD

Theresa Frangiosa, MBA

Virginia S. Biggar

Dawn M. Finnie, M.P.A.

Lauren Bangerter, PhD

Joseph E. Gaugler, PhD

Maria I. Lapid, MD

Keywords: advanced dementia, end of life, lucid intervals, caregivers

Topic: Dementia - Others

\begin{abstract}
:
People with late-stage Alzheimer's disease and related dementias (ADRD) who are assumed to have lost coherent cognitive capacity may exhibit unexpected episodes of spontaneous, meaningful, and relevant communication or behavior. Most reports of paradoxical lucidity or "episodes of lucidity" (EL) are anecdotal or case studies. Given the transient nature and lack of scientific explanation of the phenomenon, EL is under-investigated and poorly understood.
\end{abstract}

To develop an operational definition of and typologies for EL, we conducted a pilot study of former and current family caregivers from UsAgainstAlzheimer's A-LIST ${ }^{\circledR}(N=480)$. Over sixty percent of caregivers $(n=294,61 \%)$ reported witnessing at least one EL with their care recipient over the course of dementia. Most episodes happened in late stages of dementia (71\%). Only $10 \%$ happened within 7 days before death. The majority of episodes $(71 \%)$ lasted $<30$ minutes. About half the episodes were characterized by uncharacteristic speech and communication. Caregivers perceived these experiences positively $(M=$ 4.1 ; range $=1-5$ ), but also expressed desire to know why/when EL occurs and how to respond to it.

We plan to use these data to refine definitions and typologies to incorporate into a prospective, demographically diverse survey to family caregivers to assess predictors of EL and linking EL to caregiver well-being and bereavement response. Precise and robust operationalizations of EL will allow future research to assess if EL has different effects on ADRD prognosis or alters how family members understand, manage and adapt to a PLWD's dementia progression. 